\title{
Conflictos socioambientales: identificación y representación espacial. Estudio de caso en la ciudad de Río Cuarto (Argentina) Socio-environmental conflicts: identification and spatial representation. Case study of the city of Rio Cuarto (Argentina)
}

\author{
María de los Angeles Galfioni", Américo Degioanni**, \\ Gabriela Maldonado ${ }^{* * *}$ y Osvaldo Campanella*****
}

\section{INTRODUCCIÓN}

Una situación conflictiva es aquella en la que individuos con intereses contrapuestos entran en confrontación o disputa. Cuando tales acciones constituyen una lucha por valores, estatus, poder o acceso a recursos escasos se origina un conflicto social (Coser, 1956). En este sentido, cuando el conflicto es generado por la forma de uso de los recursos naturales o cuando las comunidades se ven directamente afectadas por los impactos derivados de un determinado proyecto (Orellana, 1999 — citado por Walter, 2009-) o actividad territorial (Sabattini, 1997; Sosa, 2005; Novo, 2012) se denomina conflicto socioambien-

\footnotetext{
* Departamento de Geografía, Facultad de Ciencias Humanas, Universidad Nacional de Río Cuarto (mgalfioni@hum.unrc.edu.ar).

** Departamento de Ecología Agraria, Facultad de Agronomía y Veterinaria, Universidad Nacional de Río Cuarto (adegioanni@ayv.unrc.edu.ar).

**** Departamento de Geografía, Facultad de Ciencias Humanas, Universidad Nacional de Río Cuarto. CONICET (gimaldonado@hum.unrc.edu.ar).

**** Departamento de Geología, Facultad de Ciencias Exactas, Físico-Químicas y Naturales, Universidad Nacional de Río Cuarto (ocampanella@exa.unrc.edu.ar).
} 
tal. Actualmente se evidencia que la expansión urbana genera, por un lado, la inclusión de usos de suelo que con anterioridad se localizaban en la periferia, y por otro, el encuentro con actividades vinculadas al ámbito periurbano o rural -agrícola, industrial, comercial— que confrontan con aquellas del ámbito urbano, aumentando de esta manera el campo de conflictos socioambientales. En este contexto, es el Estado —nacional, provincial, municipal— quien debe mediar para armonizar y conciliar el interés privado con el interés público, asegurando la existencia de espacios ambientalmente sanos, confortables y seguros para todos los sectores sociales sin desmedro de la actividad económica.

En función de lo enunciado en el párrafo anterior, el objetivo de este trabajo es realizar una sistematización, caracterización y cartografía de la conflictividad socioambiental en la ciudad de Río Cuarto - Argentina, provincia de Córdoba - con apoyo de un Sistema de Información Geográfica (SIG).

En lo que refiere a los antecedentes del tema, se puede citar el proyecto GEO Ciudades enmarcado dentro del Programa de las Naciones Unidas para el Medio Ambiente (PNUMA) y para el Desarrollo (2010). El mismo fue implementado, entre otros países, en Argentina, a través de estudios de evaluación de impacto ambiental en ciudades como Córdoba. En la misma línea, Scoones y Sosa (2005) presentan una compilación de trabajos sobre conflictos socioambientales en la provincia de Mendoza, originados por actividades ligadas tanto al ámbito urbano como rural, que indagan sobre sus causas y consecuencias inmediatas. Por otro lado, los reportes anuales elaborados por la Adjuntía para la Prevención de Conflictos Sociales y la Gobernabilidad de la Defensoría del Pueblo del Perú (2011) están destinados a identificar y clasificar conflictos sociales, entre ellos los socioambientales, que se constatan en el país, sobre la base de información proporcionada por los actores intervinientes. Por último, el Libro Verde de medio ambiente urbano (Ministerio de Agricultura, Alimentación y Medio Ambiente de España, 2007) es un documento que evalúa la organización y configuración de las ciudades españolas en el actual contexto y los conflictos que se derivan de los procesos intervinientes.

Los trabajos citados se presentan como una instancia de reflexión sobre los conflictos emergentes en cada uno de los espacios analizados a diferentes escalas —nacional, provincial y local—, a los fines de conciliar los intereses en contraposición dentro de un marco de participación democrática de todos los agentes intervinientes.

Si bien la conflictividad socioambiental puede ser analizada desde la perspectiva del riesgo ambiental, focalizando la atención en situaciones que potencialmente pueden generar perjuicios, o desde el estudio de impactos ambientales que determinadas acciones generaron, generan o pueden generar; 
el presente trabajo analiza la problemática sobre la base de situaciones conflictivas actuales o pasadas que se originan por la confluencia de usos de suelo que dan origen a confrontaciones y/o contradicciones socio-territoriales. Esta caracterización pretende ser un aporte para facilitar el ordenamiento territorial en donde la gestión del riesgo y la gestión ambiental son elementos gravitacionales (Grupo Internacional Recursos del Sur, 2007).

En este sentido, este trabajo procura aportar una metodología para identificar y caracterizar algunos efectos negativos, los cuáles derivan de los procesos de crecimiento urbano que se evidencian actualmente en la mayoría de las ciudades intermedias de América Latina, tomando como estudio de caso la ciudad de Río Cuarto - Córdoba, Argentina- De esta manera, a partir de la utilización de fuentes de referencias periodísticas se pretende contribuir, desde un análisis exploratorio y situado, a la identificación, clasificación y comprensión de conflictos socioambientales existentes en el área de estudio.

\section{ENCUADRE TEÓRICO-METODOLÓGICO}

La implementación y persistencia en Argentina de la doctrina neoliberal, en la que el mercado se constituye en la principal fuerza que determina y ordena las actividades que se materializan en el espacio, generan efectos territoriales no siempre compatibles entre los intereses del sector público y privado. En el marco de este escenario, las ciudades pasan a convertirse en puntos nodales de las economías internas, con un crecimiento poblacional que conlleva a un crecimiento espacial desordenado, que se materializa en la intensificación de los procesos de ocupación de tipo urbano tanto vertical como horizontal y en la confrontación de usos de suelos en el territorio, sin una ordenación integral y sostenible que armonice aspectos económicos, sociales y ambientales. Así, el interés privado, que se vincula a las fuerzas del mercado, termina direccionando las formas de crecimiento urbano que determinan una evolución que tiende a la generación de situaciones de conflictividad (Gómez Orea, 1994).

En cuanto a la forma de crecimiento urbano se reconocen principalmente dos modelos: el compacto y el difuso (Azcárate Luxán et al., 2008; Castells, 2001; Dematteis, 1998; Janoschka, 2002; Santos Preciado, 2001). No obstante, la tendencia actual es la urbanización difusa — ciudades sin centrodonde además del espacio destinado para residencias, relativamente alejado de las urbes compactas, se requiere de espacios adicionales para los servicios necesarios — comunicación, comercios, áreas de esparcimiento, etc.- lo que incrementa la demanda de lugares y por lo tanto la conflictividad. Aún así, es 
posible evidenciar nuevas lógicas de urbanización que incorporan en su estructura urbana ambos modelos, un ejemplo de esto lo constituye la ciudad de Río Cuarto (Maldonado y Campanella, 2005; Galfioni et al., 2012; De Prada et al., 2012). Como consecuencia de los expresado anteriormente, Reese (2006) indica que las transformaciones territoriales acaecidas en ciudades argentinas en los últimos 25 años, demuestran la necesidad de plantear nuevas teorías y agenda de temas a considerar para los espacios urbanos y la gestión pública derivada de los conflictos socioambientales que se están suscitando, que incluye no sólo aquellos que se producen en la estructura interna de las ciudades sino también los que se dan en su área de influencia.

Se define a un conflicto socioambiental como un proceso social y situado, en el que interactúan intereses contrapuestos producto de la materialización en el espacio de acciones individuales o colectivas, legitimadas por una racionalidad dominante y que generan pujas de poder entre sectores (Sosa, 2005). Los conflictos se expresan en formas de protestas, reclamos o disputas y se comportan como procesos dinámicos y cíclicos en el tiempo que involucran estadios de latencia, de actividad y de resolución (Walter, 2009).

Como punto de partida para el estudio de este tipo de problema, es razonable plantearse a priori interrogantes que orienten la investigación tales como ¿Qué tipos de conflictos socioambientales se evidencian?, ¿Quiénes son los agentes involucrados en dichos conflictos — demandados y demandantes-? Los conflictos identificados, ¿a qué tipo de usos de suelo se vinculan y qué efectos sobre el ambiente generan? ¿Cuál es su distribución territorial?, ¿Cuál es el marco normativo regulatorio?, ¿Qué supuestos explicativos subyacen en dicha distribución?

Para un abordaje analítico e interpretativo detallado de esta situación que permita ir dando respuesta a los interrogantes formulados, un camino metodológico conducente es el siguiente:

1. Foco y área de expresión del conflicto socioambiental, es decir la localización del mismo y el área de influencia de sus efectos. Para su delimitación se puede recurrir a la búsqueda de fuentes de referencias externas - - textos legales, trabajo empírico, experiencias en laboratorio- que permitan establecer el alcance espacial de los efectos negativos de dicho conflicto (Bosque Sedra, 2004).

2. Dinamismo o desarrollo temporal del conflicto (Walter, 2009; Sosa, 2005), se reconocen tres estadios o etapas en la que puede encontrarse un conflicto: de latencia, definida por la existencia de un problema o tema contencioso, que plantea la atención de algún sector del Estado; activo que se inicia con la formulación de una demanda por algún actor social a través acciones de protesta para solicitar una respuesta por 
parte de alguna autoridad estatal; y por último, el estadio de resolución del conflicto (Adjuntía para la prevención de conflictos sociales y la gobernabilidad de la Defensoría del Pueblo del Perú, 2011).

3. Agentes ambientales involucrados en el conflicto. Bru (1997, p. 19) los define como «todos aquellos sujetos, públicos y privados, individuales y colectivos, cuyas decisiones y acciones inciden, cualitativa y/o cuantitativamente, sobre el medio ambiente y que, a su vez, son influenciados por los cambios inducidos en él». En tal sentido, esta autora reconoce cuatro tipos de agentes: el sector privado, que agrupa a las formas y estructuras de producción; el sector público, que actúa como productor directo, suministrador de servicios y encargado de emitir las normativas y controlar su cumplimiento; las instancias técnicas-científicas ofrecidas por organismos de investigación aplicada; y, la ciudadanía, que incluye actores individuales y grupales.

4. Acciones de demanda o protesta realizadas por los agentes ambientales demandantes, las que pueden abarcar: declaraciones públicas — prensa, radio, televisión, reclamos telefónicos-, manifestaciones activas - marchas y/o cortes de vías de circulación terrestre- y demandas legales — denuncias policiales y/o juicios-.

5. Causas que explican las acciones realizadas por los agentes ambientales demandados que generan la situación de conflictividad.

6. Tipos de uso de suelo en conflicto, entendidos como «las diferentes utilizaciones que se hacen del espacio en función de las necesidades y actividades de la población que vive y trabaja en la ciudad» (Zárate, 1984, p. 60) y su área de influencia.

7. Consecuencias o efectos negativos producidos sobre la naturaleza y la sociedad que demuestren el grado de desarticulación entre ambas.

Sobre el enfoque conceptual y metodológico explicitado, se procede a la identificación, clasificación y comprensión de los conflictos socioambientales para el período 2001-2011 en la ciudad de Río Cuarto (Argentina).

\section{CARACTERIZACIÓN DE ÁREA DE ESTUdio}

El área de estudio comprende el radio municipal actual ${ }^{1}$ de la ciudad de Río Cuarto, establecido según Ley Provincial 9143 en el año 2003 con una su-

\footnotetext{
${ }^{1}$ Se entiende por radio municipal a la zona en la cual se prestan total o parcialmente los servicios públicos municipales (Municipalidad de Río Cuarto, 1996). 
FIGURA 1

LOCALIZACIÓN DEL ÁREA DE ESTUDIO

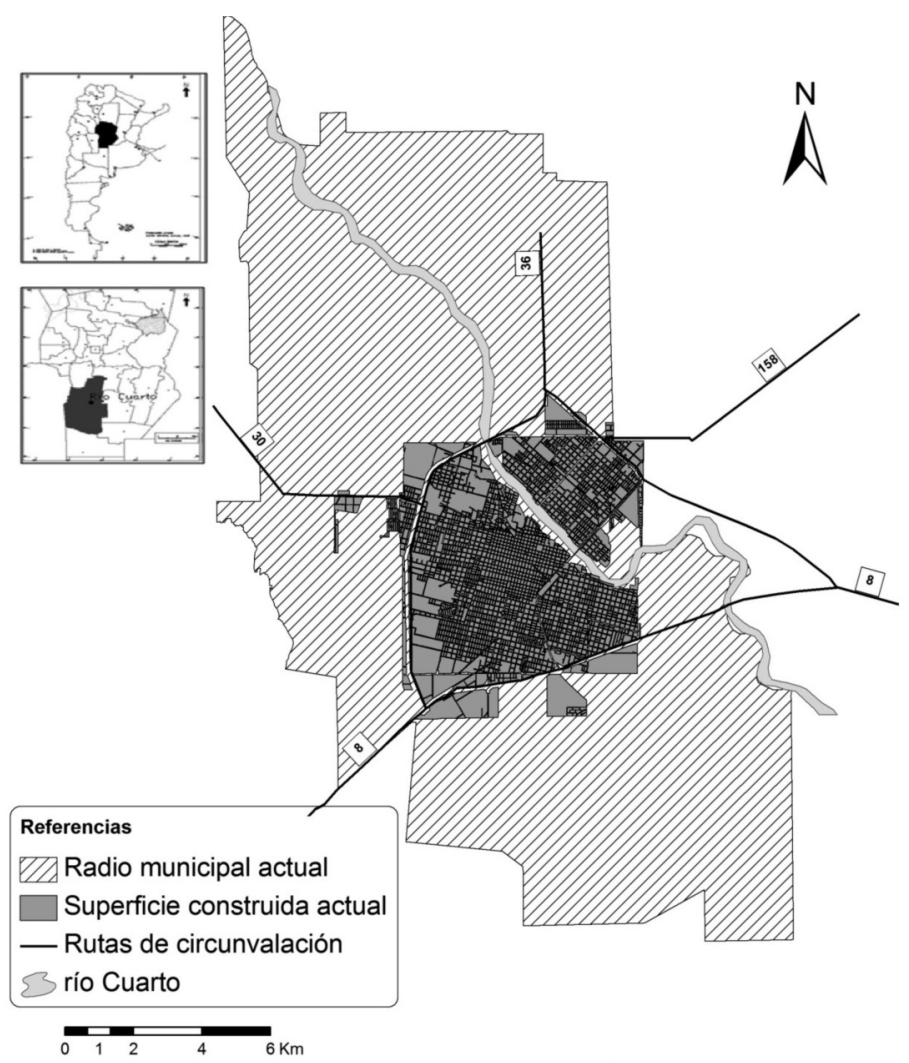

Fuente: elaboración propia (2012).

perficie de $260 \mathrm{~km}^{2}$. Este centro urbano se ubica al sudoeste de la provincia de Córdoba -Argentina—, en el departamento homónimo (figura 1).

Río Cuarto es una ciudad de tamaño intermedio con una población de 155.911 habitantes según el Censo Provincial 2008 que actualmente se concentra en una superficie de $64,25 \mathrm{~km}^{2}$. Es la segunda ciudad más importante de la provincia de Córdoba con relación a la cantidad de habitantes y servicios que ofrece - comerciales, financieros, educativos y de salud-, por lo que constituye la capital alterna provincial.

Actualmente Río Cuarto no escapa a los procesos que están ocurriendo en gran parte de los espacios urbanos argentinos, caracterizados principalmente 
FIGURA 2

\section{EXPANSIÓN DEL MEDIO CONSTRUIDO EN LA CIUDAD DE RÍO CUARTO (CÓRDOBA, ARGENTINA) ENTRE LOS AÑOS DE 1965 Y 2012}

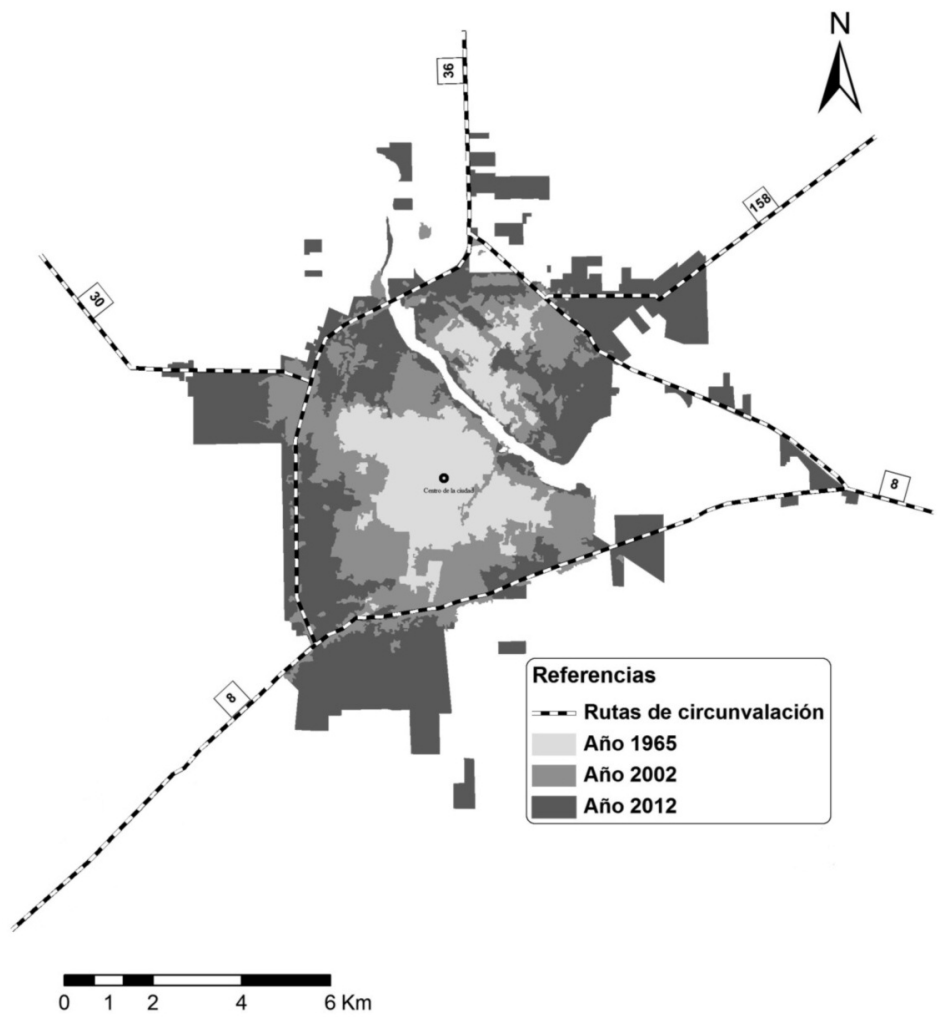

Fuente: elaboración propia en base al trabajo de Maldonado y Campanella (2004).

por la expansión urbana. A fin de observar el crecimiento del medio construido (figura 2) se tomó como referencia el trabajo realizado por Maldonado y Campanella (2004) quienes determinaron la superficie construida en 1965 y 2002, y se actualizó para el presente trabajo el año $2012^{2}$. Cuando se superpo-

2 Para los años 1965 y 2002 los autores delimitaron la superficie construida considerando y distinguiendo los espacios vacíos entre las manchas de edificación. Para el año 2012, la delimitación fue realizada sin considerar los espacios vacíos entre las manchas de edificación y, dada la continuidad espacial que reviste el medio construido, se incluyó también la localidad de Las Higueras, al noreste. 
nen los tres estados de ocupación urbana del territorio puede apreciarse que la ciudad ha tenido un crecimiento expansivo desde el centro hacia las principales vías de circunvalación de la ciudad. Se observa un crecimiento más marcado en el sector noreste y sur, y en menor medida, sobre ambas márgenes del río Cuarto y hacia el oeste de la ciudad.

El crecimiento de la ciudad combina las formas compacta y difusa, por lo que se lo puede denominar como un crecimiento de «aglutinación» (Zamorano, 1992), puesto que se apoya en las formas preexistentes de la ciudad y se expande por medio de aureolas de edificación o loteos, tanto por iniciativa privada como pública. Este crecimiento está acompañado por un aumento de la densidad de población en las áreas previamente edificadas, ya sea por construcciones en altura o por ocupación de terrenos baldíos existentes, que modifican, de hecho, la densidad edilicia urbana (Maldonado y Campanella, 2005).

\section{MATERIALES Y MÉTODOS}

Los pasos metodológicos seguidos para la consecución del objetivo del presente trabajo son los siguientes:

\section{$1^{\circ}$ Etapa. Recopilación, sistematización y análisis del material periodístico}

A través de un relevamiento propio realizado sobre fuentes de información periodística consultadas en el Archivo Histórico de la ciudad y en el diario PUNTAL S.A., se recopilaron un total de 500 artículos entre los años 2001 y 2011 de los cuales 208 se corresponden específicamente a conflictos de tipo socioambiental. Posteriormente, la información recopilada fue volcada a planillas semiestructuradas (figura 3) para su análisis, siguiendo el encuadre teórico-metodológico detallado anteriormente.

\section{$2^{\circ}$ Etapa. Tratamiento estadístico de los datos sistematizados}

En esta instancia se definieron las variables cualitativas a tener en cuenta para el tratamiento de los datos recopilados y analizados en la instancia anterior. Se procedió a un análisis bivariado de los datos en función de la relación entre variables. Para ello, se relacionaron en tablas de contingencia, por un lado, el tipo de conflicto socioambiental y la cantidad de casos identificados 
FIGURA 3

PLANILLA BASE DE ANÁLISIS DE LOS CONFLICTOS SOCIOAMBIENTALES

\begin{tabular}{|c|c|c|c|c|c|c|c|c|c|}
\hline \multicolumn{10}{|c|}{$\begin{array}{l}\text { Conflicto: } \\
\text { Tipo de uso de suelo causante del Conflicto: } \\
\text { Año: }\end{array}$} \\
\hline \multirow{2}{*}{ 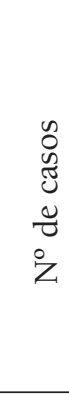 } & \multirow{2}{*}{ 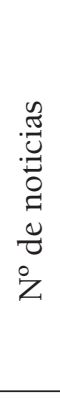 } & \multirow{2}{*}{$\begin{array}{l}\frac{\pi}{\tilde{U}} \\
\text { Uు }\end{array}$} & \multirow{2}{*}{ 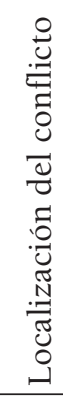 } & \multicolumn{2}{|c|}{$\begin{array}{l}\text { Agentes } \\
\text { ambientales } \\
\text { involucradas }\end{array}$} & \multirow{2}{*}{ 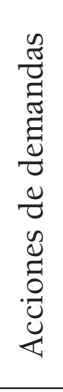 } & \multirow{2}{*}{ 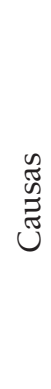 } & \multirow{2}{*}{ 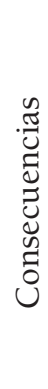 } & \multirow{2}{*}{ 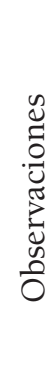 } \\
\hline & & & & 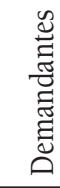 & 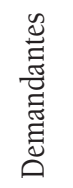 & & & & \\
\hline & & & & & & & & & \\
\hline
\end{tabular}

Fuente: elaboración propia (2012).

para cada uno de ellos entre los años 2001-2011, y por otro, la etapa de desarrollo de los conflictos socioambientales y la cantidad de casos, por tipo de conflicto, en cada una de las etapas.

\section{$3^{\circ}$ Etapa. Tratamiento cartográfico}

En primer lugar, se localizó el centro geográfico de cada conflicto. Existen dos tipos de referencias: aquellos cuya localización es exacta y los que no poseen coordenadas geográficas específicas. Para estos últimos se definió como centro geográfico un hito de referencia, como por ejemplo, un barrio o un grupo de manzanas. Luego de establecida la localización de los conflictos activos, para la determinación de su área de expresión se recurrió, en primer lugar, a los marcos normativos municipales a fin de identificar las normas que contemplan las distancias recomendadas a considerar para evitar la confluencia en el territorio de usos de suelo conflictivos. En caso de no existir regulación municipal se procedió, en segundo lugar, a la consulta de los marcos normativos provinciales, nacionales e incluso internacionales. 
En función de los marcos normativos analizados se establecieron los siguientes criterios de espacialización de los conflictos activos identificados:

a) Conflictos originados por contaminación por productos químicos y biológicos de uso industrial. Se optó por la clasificación contemplada en el manual de calidad del aire del medio urbano de la Organización Panamericana de la Salud ${ }^{3}$ (Suess et al., 1980). En este se reconocen cuatro clases de industrias con sus correspondientes áreas de influencia según el grado de peligrosidad y nocividad de las sustancias que emiten. Aplicadas al área de estudio, las mismas son:

- Ex oleaginosas y molinos harineros. Tipo II. Efectos negativos $500 \mathrm{~m}$.

- Transformadores con PCB s (cloruro de polivinilo). Tipo I. Efectos negativos $1000 \mathrm{~m}$.

- Fábrica de ladrillos. Tipo IV. Efectos negativos 100 m.

b) Conflictos originados por la presencia de basurales clandestinos. En este caso se utilizó lo estipulado por el Proyecto Nacional de Gestión Integral de Residuos Sólidos Urbanos de Argentina (2006), cuyo criterio establece una lejanía de los basurales de los asentamientos urbanos de más de $500 \mathrm{~m}$.

c) Conflictos originados por contaminación por productos químicos o biológicos de uso agropecuario. Se tomó como base la Ley Provincial 9164/04 de productos químicos o biológicos de uso agropecuario. En la misma se contemplan las áreas de protección en función de la afección ambiental derivadas de la aplicación de dichos productos según sus valores de toxicidad y residualidad, volatilidad, capacidad de percolación a napas, selectividad, concentración de producto activo y tipo de formulación. Esta ley establece la prohibición de aplicación aérea: a) dentro de un radio de $1.500 \mathrm{~m}$, desde el límite de las plantas urbanas, de productos químicos o biológicos de uso agropecuario de las clases toxicológica Ia (sumamente peligroso), Ib (muy peligroso) y III (moderadamente peligroso); y, b) dentro de un radio de $500 \mathrm{~m}$. desde el límite de las plantas urbanas, para la clase toxicológica IV. Con relación a las aplicaciones terrestres, se prohíbe el uso de productos químicos o biológicos con destino agropecuario, dentro de un radio de $500 \mathrm{~m}$., a partir del

\footnotetext{
${ }^{3}$ Dicho instrumento se utiliza como marco de referencia ya que los supuestos explicitados en el mismo son respaldados a nivel nacional por las Leyes Nacionales Ambientales $\mathrm{N}^{\circ}$ 25.612, $\mathrm{N}^{\circ} 24.051$ y No 25.670 .
} 
límite de las plantas urbanas de municipios, de las clases toxicológicas Ia, Ib y III. Sólo podrán aplicarse dentro de dicho radio los pertenecientes a la clase IV.

d) Área de conflictos generados por inundaciones. No se han registrado conflictos por inundación vinculados a crecientes del río Cuarto. Los casos detectados corresponden a inundaciones por precipitaciones abundantes e intensas. Se utilizó como criterio de demarcación el tercio inferior de cada cuenca urbana y se delimitó el área específica afectada a partir de la traza de las calles donde se identificó el conflicto.

Es de importancia destacar, que para la espacialización de los conflictos socioambientales identificados y su área de influencia adoptados en este trabajo no se consideró para ésta última el efecto gradual del daño en función de las distancias, ya que el criterio normativo - Manual de calidad del aire del medio urbano de la Organización Panamericana de la Salud, Proyecto Nacional de Gestión Integral de Residuos Sólidos Urbanos de Argentina y Ley Provincial 9164/04 de productos químicos o biológicos de uso agropecuario- utilizado para los casos identificados no considera los diferentes grados de intensidad de los efectos dentro del área expuesta. Es por tal motivo, que se asume para esta instancia de trabajo que el potencial daño causado por una actividad puede afectar de igual manera en cualquier punto del radio delimitado.

Definidos los criterios para la delimitación espacial de los conflictos socioambientales identificados, se procedió a su tratamiento en ambiente SIG, para lo cual se llevaron a cabo los siguientes pasos:

1. Confección de un modelo cartográfico como guía para la selección de la cartografía temática involucrada, a fin de definir las entidades a generar para construir el mapa de conflictividad socioambiental.

2. Selección de fuentes cartográficas de referencia provistas por el municipio de la ciudad de Río Cuarto y de ámbito científico-académico de nuestra universidad. Dicha base cartográfica comprende las entidades geográficas del manzanario de la ciudad de Río Cuarto y desagües pluviales a escala 1:20.000. Las mismas fueron proporcionadas en soporte digital en formato vectorial.

3. Introducción de información gráfica y temática al ambiente SIG, para lo cual se contemplaron las siguientes instancias:

a. Digitalización en pantalla de las capas temáticas, sobre la base de las fuentes cartográficas seleccionadas en la etapa anterior. En esta instancia se dibujaron las diferentes entidades geométricas propias del formato vectorial — puntos, polígonos y líneas-. 
b. Introducción y edición de información temática. Se crearon y/o modificaron los campos de atributos correspondientes a las capas temáticas generadas en la instancia anterior.

4. Creación de entidades referidas a la espacialización de los conflictos. Se realizaron operaciones propias de un SIG: búsqueda selectiva, superposiciones, digitalización y buffer con la finalidad de generar las áreas de influencia de los tipos de conflictos identificados.

5. Presentación y salida de información cartográfica.

\section{RESULTADOS}

\section{a) Identificación y caracterización de los conflictos socioambientales}

Con relación a los tipos de conflictos socioambientales identificados en el período 2001-2011 (tabla 1), y suponiendo objetividad en la información periodística, se evidencia que la variación interanual de casos detectados es bastante fluctuante, concentrándose sobre todo entre los años 2002-2004. Se observa un claro predominio de conflictos originados por inundaciones, y sigue en importancia, los originados por la presencia de basurales clandestinos y por último, la contaminación por productos químicos y biológicos de uso industrial y agropecuario. No obstante, se destaca una distribución temporal uniforme de los mismos. En cuanto a los otros tipos de conflictos, los números de casos identificados fueron menores y discontinuos en el tiempo.

Con referencia al estadio temporal en el que se encuentran los casos identificados (figura 4), por un lado, se detecta que en los conflictos por basurales clandestinos, inundaciones, contaminación por productos químicos y biológicos de uso agropecuario e industrial, los casos activos superan a los latentes y resueltos. Esto indica que son tipos de conflictos que continuamente se encuentran en instancia de reclamos por parte de algún agente ambiental involucrado. Por otro lado, con relación a los conflictos de contaminación por actividad pecuaria concentrada, contaminación por efluentes cloacales, erosión del río Cuarto, extracción de áridos y napas freáticas altas, la mayoría de los casos identificados se encuentran en la etapa de latencia, es decir que se produjo una disminución o desaparición de los reclamos pero aún no se ha dado una solución definitiva a los mismos. Con respecto a los casos resueltos, predomina la recurrencia en conflictos derivados de la contaminación por 


\begin{tabular}{|c|c|c|c|c|c|c|c|c|c|c|c|c|}
\hline & 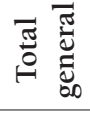 & શे & $\exists$ & $\Lambda$ & $\vec{\sim}$ & $\stackrel{\infty}{m}$ & $\infty$ & $\sim$ & 0 & $\tilde{b}$ & $\underset{\sim}{\sim}$ & $\stackrel{\infty}{\stackrel{\nu}{\nu}}$ \\
\hline 必 & ప্] & $m$ & & -1 & $m$ & $\sim$ & & & - & $\stackrel{\circ}{\circ}$ & & 귀 \\
\hline$\stackrel{0}{\tilde{0}}$ & $\stackrel{0}{\circ}$ & $\sim$ & & - & $m$ & $m$ & & & & + & & $\stackrel{m}{\sim}$ \\
\hline 宲 & 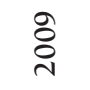 & $n$ & & & $\sim$ & $\sim$ & & & & $m$ & & $\stackrel{-}{\circ}$ \\
\hline 灾 & 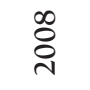 & $\neg$ & & & - & in & & $\neg$ & N & $\exists$ & $m$ & $\stackrel{ \pm}{\sim}$ \\
\hline 空 & 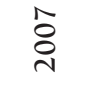 & $\sim$ & -1 & & $\sim$ & N & & $\dashv$ & & - & & $a$ \\
\hline 용 & ¿̊ & 0 & & -1 & - & $\sim$ & N & & - & $\neg$ & -1 & $\stackrel{n}{n}$ \\
\hline 定点 & $\stackrel{n}{8}$ & $\sim$ & $\neg$ & & N & + & & & - & $\wedge$ & $\sim$ & 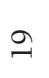 \\
\hline 己 & 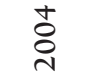 & 0 & in & & + & + & $\neg$ & & - & $\neg$ & $\sim$ & $\stackrel{+}{\sim}$ \\
\hline 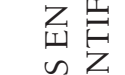 & 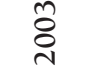 & 0 & $m$ & $\neg$ & -1 & $\infty$ & $\neg$ & & & in & $\sim$ & $\hat{\sim}$ \\
\hline 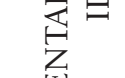 & స్ & in & - & $m$ & $N$ & $m$ & + & & & 근 & & $\stackrel{n}{m}$ \\
\hline$\sum_{i}^{E}$ & ¿̊ & $m$ & & & & $\sim$ & & & & in & $\sim$ & $\underset{-}{\sim}$ \\
\hline 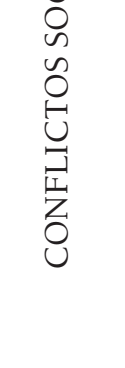 & 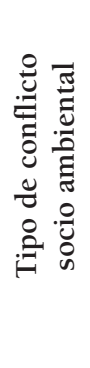 & 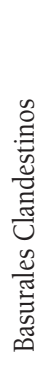 & 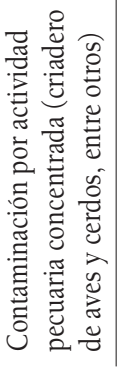 & 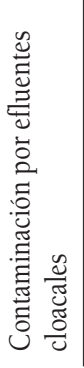 & 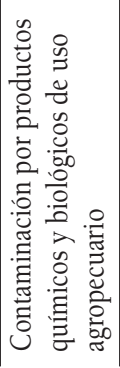 & 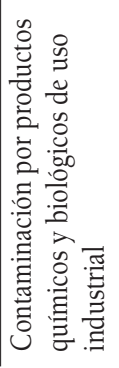 & 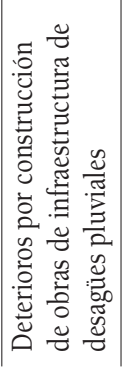 & 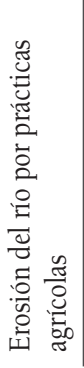 & 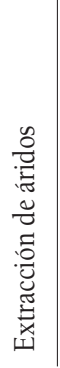 & 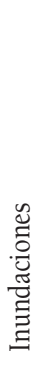 & 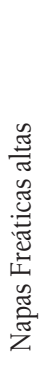 & 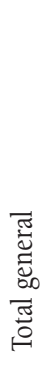 \\
\hline
\end{tabular}

Estudios Geográficos, Vol. LXXIV, 275, pp. 469-493, julio-diciembre 2013 ISSN: 0014-1496, eISSN: 1988-8546, doi: 10.3989/estgeogr.201317 
FIGURA 4

DISTRIBUCIÓN DE LOS CASOS POR TIPO CONFLICTO SOCIOAMBIENTAL SEGÚN ETAPA DE DESARROLLO EN LA CUAL SE ENCUENTRA (2001-2011)

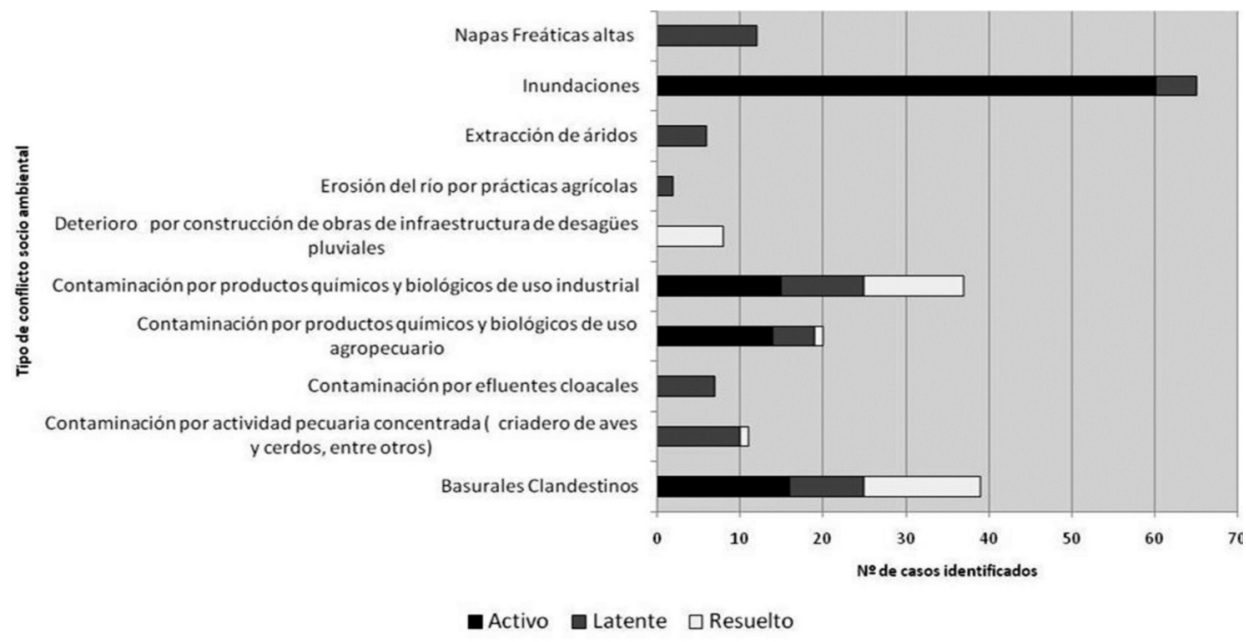

Fuente: elaboración propia, 2012.

productos químicos y biológicos de uso industrial, de la instalación de basurales clandestinos y de deterioro por construcción de desagües pluviales. En este último caso, los conflictos fueron resueltos en la instancia judicial iniciada por los agentes damnificados.

Del análisis realizado se desprende que los principales conflictos socioambientales existentes en el área de estudio son aquellos vinculados a las inundaciones, seguidos por la contaminación por productos químicos y biológicos de uso industrial y agropecuario y basurales clandestinos.

En primera instancia, con relación a los agentes ambientales demandados por tipo de conflicto, en el caso de los basurales clandestinos e inundaciones el denominador común es el sector público, el cual incluye tanto al municipio como otros entes provinciales descentralizados que se encargan del control, regulación, mantenimiento y reparación de la infraestructura. En los casos de contaminación por productos químicos y biológicos de uso agropecuario, los agentes demandados están vinculados al sector privado rural representado por 
los productores agrícolas y frutihortícolas. En los casos vinculados a la contaminación por productos químicos y biológicos de uso industrial se demanda fundamentalmente al sector privado industrial —molinos harinero, aceiteras y ladrilleras—, seguido por el sector público representado por el municipio y entes descentralizados provinciales tales como la Empresa Provincial de Energía de Córdoba, fuertemente cuestionada por su responsabilidad en el control de transformadores con PCB s.

En una segunda instancia, con referencia a los agentes ambientales demandantes en las situaciones conflictivas detectadas, en todos los tipos de conflictos aparece el predominio de la ciudadanía, siguiendo la instancia técnicas-científicas representada por diferentes profesionales del ámbito de las Universidades Nacionales de Río Cuarto y Córdoba. En muy poca proporción, se observan reclamos del sector privado rural representados por productores hortícolas que reclaman sobre los efectos negativos que producen las fábricas de ladrillos sobre su producción.

En cuanto a las acciones de demanda realizadas por los diferentes agentes ambientales implicados en los distintos tipos de conflictos, en todos los casos se destaca el predominio de las declaraciones públicas en los medios de comunicación, seguido por demandas legales en los conflictos por contaminación por productos químicos y biológicos de uso industrial y agropecuario y, por último, manifestaciones activas con cortes de rutas en los reclamos por inundaciones.

En el cuadro 1 se sintetizan las principales causas y consecuencias de los tipos de conflictos socioambientales con mayoría de casos activos identificados en la ciudad de Río Cuarto.

En síntesis, los conflictos socioambientales activos identificados y analizados en el presente apartado, que han mantenido mayor frecuencia en el período considerado, derivan de las demandas públicas iniciadas, fundamentalmente, por la ciudadanía ante los efectos negativos de las acciones que ejercen, por un lado, el sector privado a través de los usos de suelo agrícola, frutihortícola e industrial y, por otro, del sector público representado por el municipio y otros entes provinciales descentralizados cuestionados en lo que respecta a su rol como asegurador del bienestar de la población en general.

De esta manera, dichos efectos negativos no sólo generan consecuencias sobre los componentes de la naturaleza sino también sobre diferentes sectores de la sociedad que ven amenazado el ambiente en el cual habitan y construyen. 


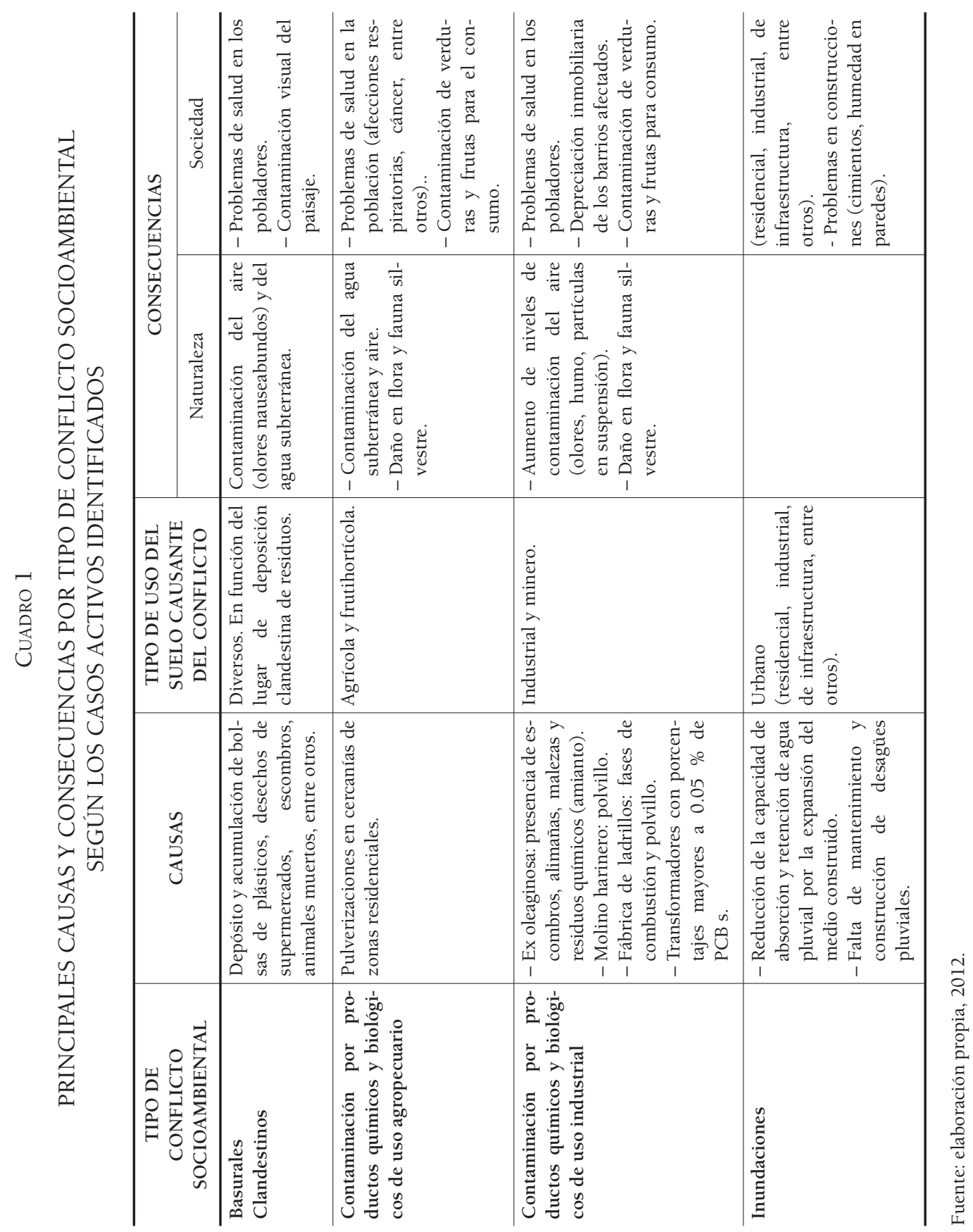

Estudios Geográficos, Vol. LXXIV, 275, pp. 469-493, julio-diciembre 2013 ISSN: 0014-1496, eISSN: 1988-8546, doi: 10.3989/estgeogr.201317 


\section{b) Distribución espacial de los conflictos socioambientales activos} identificados en el área de estudio

En la figura 5 se observan las áreas de conflictividad socioambiental en la ciudad de Río Cuarto, obtenidas a través de su tratamiento en ambiente SIG a partir de los criterios de espacialización establecidos. Se destacan dos grandes sectores: uno de conflictos en el área estrictamente urbana y otro en el área periurbana, al oeste de la ciudad.

\section{FIGURA 5}

\section{ÁREAS DE CONFLICTIVIDAD SOCIOAMBIENTAL EN LA CIUDAD DE RÍO CUARTO (CÓRDOBA, ARGENTINA)}

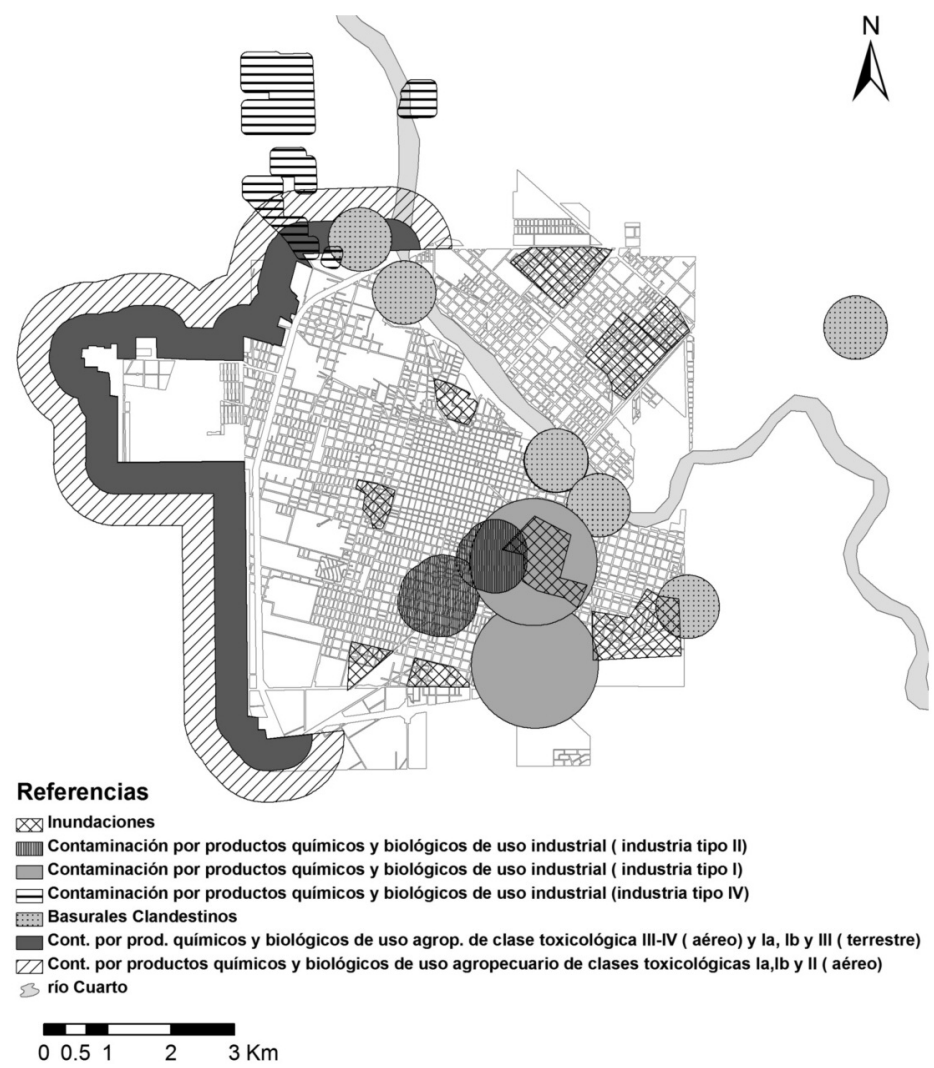

Fuente: elaboración propia. 
El conflicto que presenta mayor dispersión espacial es el derivado de las inundaciones por precipitaciones locales producto de la impermeabilización del suelo causa de la expansión del medio construido y la carencia de un sistema de desagüe eficiente. En el sector sur de la ciudad, se concentran los derivados de la actividad industrial y servicios eléctricos. En el mismo lugar, se observa una superposición de conflictos y áreas de influencia, por lo que se trata del área de mayor complejidad en lo que respecta a las conflictividades estudiadas. Por último, las áreas vinculadas a la presencia de basurales, se localizan en las márgenes del río Cuarto, de forma coincidente con asentamientos urbanos informales.

En el sector oeste de la ciudad, en el límite del área construida, es donde se han registrado la mayoría de los conflictos por contaminación por productos químicos y biológicos de uso agropecuario conjuntamente con los derivados de las fábricas de ladrillo, siendo el sector con la situación periurbana más compleja.

\section{c) El crecimiento urbano en Río Cuarto y su relación con los conflictos socioambientales identificados}

A partir de la cartografía de conflictos socioambientales realizada es posible obtener un mapa de recurrencia espacial de los mismos. Al articular la conflictividad espacial con las manchas de crecimiento urbano reconocidas para los años 1965, 2002 y 2012 (figura 6), se observa claramente que los espacios de conflictividad mencionados se vinculan a dicho crecimiento. El sector centro-este representa conflictos vinculados a un uso de suelo industrial pretérito, que en general se encuentra abandonado y que respondió a una forma de uso del territorio articulado a las vías del ferrocarril y que, por lo tanto, se localiza en los sectores más antiguos de la ciudad. Los conflictos localizados en el sector sureste de la ciudad, corresponden a la segunda etapa de expansión presentada anteriormente (año 2002), en la cual ya existía un desplazamiento de las actividades industriales hacia este sector y un notable incremento del medio construido en áreas propensas a inundaciones por la deficiencia estructural del sistema de desagüe pluvial urbano. El sector de conflictos ubicado al oeste y norte de la ciudad constituye un nuevo tipo de conflictividad que, fundamentalmente, se expresa en el espacio periurbano y es producto esencialmente de la presión que genera la expansión del espacio urbano sobre el espacio rural y viceversa. 


\section{FIGURA 6}

RECURRENCIA ESPACIAL DE CONFLICTIVIDAD SOCIOAMBIENTAL Y EXPANSIÓN DEL MEDIO CONSTRUIDO EN LA CIUDAD DE RÍO CUARTO (CÓRDOBA, ARGENTINA)

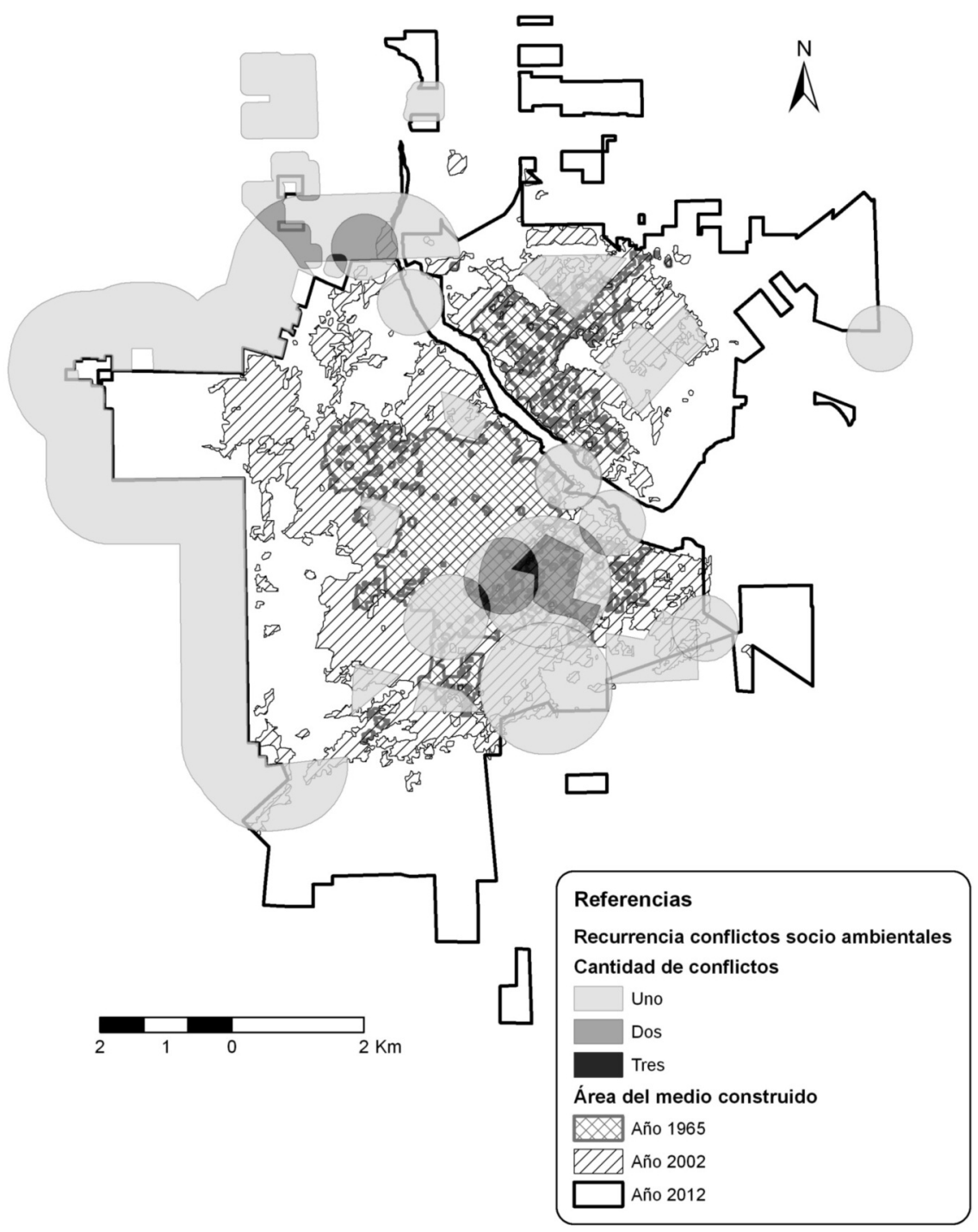

Fuente: elaboración propia en base a Maldonado y Campanella (2004), trabajo de campo y recopilación periodística. 


\section{DISCUSIÓN Y CONCLUSIONES}

Del relevamiento periodístico realizado y su posterior sistematización, se pudo reconocer que las contradicciones emergentes de los diferentes usos del suelo que generan conflictos terminan expresándose en forma de reclamos por parte de los distintos sectores que se ven directa o indirectamente perjudicados con los efectos negativos derivados. En tal sentido y de acuerdo al mapa de conflictos elaborado, se constata que, en el área urbana, existe una deficiente regulación del excedente hídrico, lo que indica que se ha permitido usar el suelo para instalar edificaciones en sitios propensos a inundarse, sin las obras de control requeridas. Algo similar ocurre con la instalación de actividades de riesgo de contaminación por diversas fuentes ya que no se han respetado las normas vigentes. En lo que respecta a los conflictos por presencia de basurales, los resultados obtenidos señalan la urgencia de erradicar las causas de su presencia por sobre la urgencia de destinar otras áreas para la deposición final de los desechos que estén fuera del área urbana. En cuanto al sector donde confluyen los límites del radio municipal antiguo y actual, emergen claramente conflictos por la tensión que genera el uso del suelo residencial y el agropecuario o minero. Al analizar la articulación entre los conflictos y la expansión urbana, se observa que en la actualidad el centro urbano y su área de influencia han ido acumulando situaciones conflictivas derivadas de usos de suelo incompatibles sin lograr resolverlas mediante la incorporación clara de las limitaciones en el marco normativo municipal y su posterior reglamentación y aplicación.

En lo que respecta a la propuesta metodológica presentada se considera que, por un lado, constituye un marco que orienta la recopilación y posterior tratamiento de la información a fin de alcanzar un documento cartográfico que contenga la expresión espacial de los conflictos socioambientales y, por otro lado, sirve de insumo para correlacionar estos resultados con otras fuentes de información vinculadas a los procesos interactuantes en el espacio urbano y su área de influencia, tales como estudios de expansión urbana, caracterización socio-económica de la población, estudios geohidrológicos, caracterización de usos de suelo, entre otros. Asimismo, se sostiene que detectar los tipos de usos de suelo conflictivos en la actualidad sirve de herramienta para prevenir situaciones semejantes derivadas del proceso de urbanización actual y futuro.

Los resultados obtenidos en el presente trabajo aportan información relevante sobre el estado de situación de la conflictividad socioambiental en la ciudad de Río Cuarto para la planificación y gestión sobre el espacio, identifi- 
cando los problemas vigentes y aportando una visión espacial que permite priorizar acciones que busquen soluciones de compromiso entre los diferentes actores, para la configuración de espacios seguros y saludables para la población que tiendan a un equilibrio territorial en el marco de un proceso continuo de crecimiento urbano sostenible.

\section{AgradeCIMIENTOS}

Agencia Nacional de Promoción Científica y Técnica —-Ministerio de Ciencia y Tecnología de la Provincia de Córdoba- y BID-PID No 013/2009.

Fecha de recepción: 25/05/2012

Fecha de aceptación: 22/02/2013

\section{BiBLIOGRAFÍA}

Azcárate Luxán, M. V., Cocero Matesanz, D., Fernández, A., García Lázaro, F. J., Muguruza Cañas, C. y Santos Preciado, J. M. (eds.) (2008): "Rasgos fundamentales del reciente proceso de urbanización difusa. Algunas reflexiones sobre la realidad de la ciudad dispersa en las aglomeraciones urbanas españolas”, en M. Á. Díaz, J. Bosque y R. Mata (coords.): XI Coloquio Ibérico de Geografía. La perspectiva geográfica ante los nuevos retos de la sociedad y el medio ambiente en el contexto ibérico., Alcalá de Henares, 1-4 de octubre de 2008. Departamento de Geografía - Universidad de Alcalá, Asociación de Geógrafos Españoles (AGE) y Asociación Portuguesa de Geógrafos (APG). Disponible en: http://www.geogra.uah.es/web_11_cig/cdXICIG/docs/01-PDF_Comunicaciones_coloquio/pdf-1/com-P1-24.pdf (Fecha de consulta: 16/07/2013).

Bosque Sendra, J., Díaz Castillo, C., Díaz Muñoz, M. A., Gómez Delgado, M., Gónzalez Ferreiro, D., Rodríguez Espinosa, V. M. y Salado García, M. J. (2004): "Propuesta metodológica para caracterizar las áreas expuestas a riesgos tecnológicos mediante SIG. Aplicación en la Comunidad de Madrid”. Revista GeoFocus, 4, pp. 44-78.

Bru, J. (1997): Medio ambiente, poder y espectáculo. Barcelona, Editorial ICARA.

Castells, M. (2001): La galaxia Internet. Barcelona, Editorial Areté.

Coser, L. A. (1956): The Functions of Social Conflict. London, New York and CollierMacmillan.

De Prada, J., Degioanni, A., Cisneros, J., Galfioni, M. y Cantero, A. (2012): “Diseño y evaluación de propuestas de ordenamiento de territorio: La urbanización sobre tierras rurales", en: XLIII Reunión Anual de la Asociación Argentina de Economía Agraria. Argentina, Corrientes, pp. 1-32. 
Defensoría del Pueblo (ed.) (2011): "Adjuntía para la Prevención de Conflictos Sociales y la Gobernabilidad". Reportes de Conflictos Sociales, 89. Disponible en: http://www.congreso.gob.pe/dgp/didp/boletines/CARPETA_ICA/Ica_FORSUR/De fensor\%C3\%ADa\%20del\%20Pueblo/Reporte-89-2011_ReportedeConflictosSo ciales.pdf (Fecha de consulta: 16/07/2013).

Dematteis, G. (1998): "Suburbanización y periurbanización. Ciudades anglosajonas y ciudades latinas", en. J. Monclús (ed): La ciudad dispersa: suburbanización y nuevas periferias. Barcelona, Centro de Cultura Contemporánea, pp. 17-34.

Galfioni, M. A., Maldonado, G. y Degioanni, A. (2012): “Ciudad, expansión del medio construido, conflictos y contradicciones. Estudio de caso de la ciudad de Río Cuarto (Pcia. de Córdoba)", en: 6 Coloquio geográfico sobre América Latina. "Las nuevas configuraciones territoriales latinoamericanas desde una perspectiva geográfica”, Paraná 14-17 marzo. Santa Fe, Universidad Nacional del Litoral, Departamento de Geografía de la Facultad de Humanidades, Artes y Ciencias Sociales, pp. 1-11.

Gómez Orea, D. (1994): Ordenación del territorio. Madrid, Editorial Agrícola Española S.A. e Instituto Tecnológico Geo-Minero de España.

Grupo Internacional Recursos del Sur (ed.) (2007): Tiempo para entregar el relevo: reducción del riesgo de desastres desde la perspectiva de la gestión ambiental, ordenamiento territorial, finanzas e inversión pública. Costa Rica, San José Editorial.

Janoschka, M. (2002): "El nuevo modelo de la ciudad latinoamericana: fragmentación y privatización". Revista Eure, 28/85, pp. 11-30.

Maldonado, G. y Campanella, O. (2004): "Evolución de la Mancha Urbana de la ciudad de Río Cuarto, Córdoba, Argentina, mediante la aplicación de Tecnología de Sensoramiento Remoto y Sistemas de Información Geográfica”, en 6 Encuentro Internacional Humboldt. Córdoba, Villa Carlos Paz, pp. 1-11.

Maldonado, G. y Campanella, O. (2005): "Tendencia de crecimiento urbano y medio físico en la ciudad de Río Cuarto, Córdoba. Argentina”, en B. Villegas, E. Laurelli y G. Fasciolo (eds.): III Seminario Internacional "La interdisciplina en el Ordenamiento Territorial”. Mendoza, Instituto de Cartografía, Investigación y Formación para el Ordenamiento Territorial, y Facultad de Filosofía y Letras de la Universidad Nacional de Cuyo, pp. 1-15.

Ministerio de Agricultura, Alimentación y Medio Ambiente (ed.) (2007): Libro Verde de medio ambiente urbano. Madrid, Ministerio de Agricultura, Alimentación y Medio Ambiente. Disponible en: http://bcnecologia.net/es/proyectos/libro-verde-demedio-ambiente-urbano-tomo-i-y-ii (Fecha de consulta: 16/07/2013).

Novo Martínez, R. (2012): "Un modelo de análisis del conflicto socio-ambiental para aprender-investigar". Revista Latinoamericana de Metodología de la Investigación Social, 33/2-2, pp. 35-46.

Orellana, R. (2009): "Conflictos... ¿Sociales, ambientales, socioambientales? ... conflictos y controversias en la definición de los conceptos", en M. Walter (ed.): "Conflictos ambientales, socioambientales, ecológico distributivos, de contenido ambiental. Reflexionando sobre enfoques y definiciones". Boletín ECOS, 6/febrero-abril, p. 2. 
Programa de las Naciones Unidas sobre el Medio Ambiente (PNUMA) (2010): Perspectivas del medio ambiente: Geo Córdoba. Córdoba, PNUMA. Disponible en: http://www.pnuma.org/deat1/pdf/2010\%20-\%20GEO\%20Cordoba.pdf. (Fecha de consulta: 16/07/2013).

Republica Argentina. "Proyecto Nacional de Gestión Integral de Residuos Sólidos Urbanos Préstamo BIRF 7362-ARLPI-0-7". Disponible en: http://www.ambiente. gob.ar/rsu 2012. 2006 (Fecha de consulta: 16/07/2013).

Reese, E. (2006): "La situación actual de la gestión urbana y la agenda de las ciudades en la Argentina". Medio Ambiente y Urbanización, 6, pp. 3-22.

Sabattini, F. (1997): "Conflictos ambientales y desarrollo sustentable de las regiones urbanas". Revista EURE, 23/68, pp. 77-91.

Santos Preciado, J. M. (2001): "Las periferias urbanas y la organización de la ciudad actual: el caso de Madrid". Ciudad y Territorio. Estudios Territoriales, 126, pp. 669- 688.

Scoones, A. y Sosa, E. (comp.) (2005): Conflictos socio ambientales y políticas públicas en la provincia de Mendoza. Mendoza, Oikos Red Ambiental.

Sosa, E. (2005): "Los Conflictos socio ambientales en la provincia de Mendoza: Marco conceptual", en A. Scoones y E. Sosa (comp.): Conflictos socio ambientales y políticas públicas en la provincia de Mendoza. Mendoza, Oikos Red Ambiental, pp. 204-214.

Suess, M. J. y Craxford, S. R. (1980): Manual de calidad del aire del medio urbano. Organización Panamericana de la Salud. Ginebra, Organización Mundial de la Salud, Organización Panamericana de la Salud.

Walter, M. (2009): “Conflictos ambientales, socioambientales, ecológico distributivos, de contenido ambiental. Reflexionando sobre enfoques y definiciones". Boletín ECOS, 6/febrero-abril.

Zamorano, M. (1992): Geografía urbana. Formas, funciones y dinámica de las ciudades. Colección Geográfica. Argentina, Editorial Ceyne.

Zarate, A. (1984): Mosaico Urbano. Madrid, Editorial Cincel S.A.

\section{LEGISLACIÓN}

Republica Argentina. Ley Provincial no 9164, Productos químicos o biológicos de uso agropecuario, Ministerio de Agricultura, Ganadería y Alimento. Disponible en: http://magya.cba.gov.ar/upload/Ley_9164_Productos_Quimicos_y_Biologicos_de_ Usos_Agropecuarios.pdf (Fecha de consulta: 16/07/2013).

Republica Argentina. Municipalidad de la ciudad de Río Cuarto, Carta Orgánica Municipal, 1996. Disponible en: http://www.mininterior.gov.ar/municipios/archivos_ regimen/CO_CBA_rio_cuarto.pdf (Fecha de consulta: 16/07/2013). 


\section{RESUMEN}

En la ciudad de Río Cuarto (provincia de Córdoba, Argentina) se advierte en la actualidad que el proceso de expansión urbana genera, por un lado, la inclusión de usos de suelo que con anterioridad se localizaban en la periferia, y por otro, el encuentro con actividades vinculadas al ámbito periurbano o rural (agrícola, industrial, comercial) que confrontan con aquellas del ámbito urbano, aumentando de esta manera el campo de conflictos socio ambientales. Esta situación, obliga al ámbito científico a generar conocimientos que sirvan para la toma de decisiones por parte del Estado, que debe conciliar intereses contrapuestos y asegurar un desarrollo sostenible. Por tratarse de conflictos con una fuerte implicancia territorial, el abordaje de los mismos se hace a partir de una cartografía actualizada y acorde a los objetivos propuestos. El objetivo del presente artículo es elaborar una sistematización, caracterización y posterior cartografía de conflictividad socio ambiental de la ciudad de Río Cuarto en un entorno SIG. Para vincularla luego con el proceso de expansión del medio construido urbano.

Palabras Clave: crecimiento urbano; usos de suelo; conflictos socio ambientales; agentes ambientales; Sistema de Información Geográfica.

\section{ABSTRACT}

The city of Rio Cuarto (Cordoba Province, Argentina) currently provides clear indications that the process of urban sprawl generates, on one hand, uses of land that were previously located in the periphery, and secondly, collisions with the activities of suburban or rural areas (agricultural, industrial, commercial) which find themselves coming up against the activities of urban areas, thus expanding the field of socio-environmental conflict. Such a situation obliges scholars to generate knowledge for use in the making of decisions by the State, whose task it is to reconcile conflicting interests and ensure sustainable development. Given that such conflicts haveclear territorial implications, all attempts to address them must be based on up-to-date mapping projects and be consistent with perceived objectives. The aim of this paper is thus to carry out systematic characterization and mapping of environmental social unrest in the city of Rio Cuarto in a GIS environment. The paper then links such unrest with expansion of the built-up urban environment.

In the city of Rio Cuarto (Cordoba Province, Argentina) today warns that the process of urban sprawl generates, on one hand, the inclusion of land uses that previously were located in the periphery, and secondly, the encounter with activities related to suburban or rural area (agricultural, industrial, commercial) faced with those from urban areas, thus increasing the field of socio-environmental conflicts. This situation forces the scientific field to generate knowledge for use in the making of decisions by the State that is who must reconcile conflicting interests and ensure sustainable development. Because it conflicts with a strong territorial implications, addressing them is a mapping from updated and consistent with the objectives. The aim of this paper is to develop a systematic, characterization and subsequent mapping of environmental 
social unrest in the city of Rio Cuarto in a GIS environment. To link it then with the expansion of the urban built environment.

KEY WORDS: urban growth; land use; socio-environmental conflicts; environmental agents; GIS.

\section{RÉSUMÉ}

Dans la ville de Río Cuarto (province de Cordoba, Argentine) on apprécie aujourd'hui que le processus d'expansion urbaine donne lieu, d'une part, à des usages du sol qui étaient auparavant localisés dans la périphérie et, d'autre part, à un choc entre les activités liées à la zone suburbaine ou rurale (agricoles, industrielles, commerciales) et celles des zones urbaines, ce qui augmente le champ de conflits socio-environnementaux. Cette situation oblige les disciplines scientifiques à produire des connaissances servant à la prise de décisions de la part de l'État qui doivent concilier des intérêts contradictoires et assurer un développement durable. Étant donné qu'il s'agit de conflits avec de fortes implications territoriales il faut les aborder à partir d'une cartographie mise à jour et conforme aux objectifs envisagés. Le but de cet article est de développer une systématisation, une caractérisation et une postérieure cartographie des conflits socio-environnementaux dans la ville de Río Cuarto dans un entourage SIG. Cela doit être par la suite mis en relation avec l'expansion du milieu urbain construit.

MoTS CLÉS: croissance urbaine; usages du sol; conflits : croissance urbaine; usages du sol; conflits socio-environnementaux; agents environnementaux; Système d'Information Geographique.o-environnementaux; agents environnementaux; Système d'Information Geographique. 